TRANSACTIONS OF THE

AMERICAN MATHEMATICAL SOCIETY

Volume 359, Number 12, December 2007, Pages 6167-6186

S 0002-9947(07)04357-7

Article electronically published on July 23, 2007

\title{
ENTROPY POINTS AND APPLICATIONS
}

\author{
XIANGDONG YE AND GUOHUA ZHANG
}

\begin{abstract}
First notions of entropy point and uniform entropy point are introduced using Bowen's definition of topological entropy. Some basic properties of the notions are discussed. As an application it is shown that for any topological dynamical system there is a countable closed subset whose Bowen entropy is equal to the entropy of the original system.

Then notions of C-entropy point are introduced along the line of entropy tuple both in topological and measure-theoretical settings. It is shown that each C-entropy point is an entropy point, and the set of C-entropy points is the union of sets of C-entropy points for all invariant measures.
\end{abstract}

\section{INTRODUCTION}

Entropy is defined in both ergodic theory and topological dynamical systems (TDS for short). Starting with the study of the topological analogue of Kolmogorov systems, in recent years much attention has been paid to the so called local properties of entropy and its many interesting results. First Blanchard [2] introduced the notion of an entropy pair in topological dynamics from which the existence of the maximal zero entropy factor of a given topological system was proved ([3]), and Blanchard et al. 5] defined the notion of an entropy pair for an invariant measure which naturally led to the discussion of the relation between the two kinds of entropy pairs. Then Huang and Ye 15] introduced the notion of entropy tuples both in topological and measure-theoretical settings. Just recently, Dou, Ye and Zhang [9] generalized the notion (of entropy tuples) to entropy sequences and entropy sets in both settings. To study the relation of entropy tuples in both settings, a local variational inequality [4, a local variational relation [15] and finally local variational principles 20, 14] which refine the classical variational principle were found. Moreover, a local variational principle for conditional entropy was proved [16], and relative entropy tuples in both settings, relative u.p.e. and c.p.e. extensions were studied [17.

The main purpose of the paper is to fill a missing case: entropy point. First several notions of entropy points, namely, entropy point, full entropy point, uniform entropy point and uniform full entropy point are introduced using Bowen's definition of topological entropy. Some basic properties of them are discussed. It turns out that there are no entropy points of any kind if we consider a TDS with

Received by the editors September 15, 2005 and, in revised form, May 15, 2006.

2000 Mathematics Subject Classification. Primary 37A05, 37A35.

Key words and phrases. Topological entropy, entropy for a measure, entropy tuple, entropy point.

Both authors were partially supported by Ministry of Education (no. 0358053), and the first author was partially supported by NSFC (no. 10531010).

(c)2007 American Mathematical Society Reverts to public domain 28 years from publication 
zero entropy, and the support of any ergodic measure with positive entropy is contained in the set of uniform entropy points. In general, the sets of entropy points by the mentioned four definitions are different, and they coincide with each other and equal the whole space if we consider a minimal TDS with positive entropy. A somewhat surprising consequence of the study of uniform entropy points is that for any topological dynamical system there is a countable closed subset whose entropy is equal to the entropy of the original system. In fact, the countable subset $A$ can be chosen such that the set of the limit points of $A$ has at most one limit point. It is worth mentioning that some applications of the above results will be carried out in a forthcoming paper in [18].

Second along the line of entropy pair, tuple, sequence and set, the notions of Centropy point are introduced both in topological and measure-theoretical settings. It is shown that each C-entropy point is an entropy point, and the set of C-entropy points is the union of sets of C-entropy points for an invariant measure over all invariant measures. However, even if we consider a minimal TDS with positive entropy, the set of C-entropy points need not be the whole space.

After finishing the writing of the paper, we found that in [10] the authors also introduced a notion called entropy point when studying preimage entropy of continuous maps, which measures the degree of the non-invertibility of the system. Thus, their notion is essentially different from our's. Moreover, we note that in a forthcoming paper by Blanchard and Huang [6], the notion of C-entropy point is introduced in a different way.

The paper is organized as follows. In section 2 after stating some basic properties of Bowen's entropy, we introduce notions of entropy point and full entropy point. In section 3 we study the structure of the set of entropy points and prove that the support of any ergodic measure with positive entropy is contained in the set of entropy points. In section 4 we define and study entropy function serving as a tool of studying uniform entropy point. In section 5 by means of entropy function we introduce and discuss some properties of uniform entropy point and uniform full entropy point, and show that for any topological dynamical system there is a countable closed subset whose entropy is equal to the entropy of the original system. Finally in section 6 along the line of entropy tuple we introduce notions of C-entropy point both in topological and measure-theoretical settings, and investigate some basic properties of them.

\section{ENTROPY POINT AND FULL ENTROPY POINT}

In this section after recalling Bowen's definition of topological entropy, we introduce and discuss some basic properties of entropy point and full entropy point.

By a topological dynamical system we mean a pair $(X, T)$, where $X$ is a compact metric space and $T: X \rightarrow X$ is a self-homeomorphism. Before proceeding, let's first recall Bowen's definition of topological entropy using separated and spanning sets and some relevant results (see [21], $\left.\mathrm{P}_{168-174}\right)$.

Let $d$ be a metric on TDS $(X, T)$. For each $n \in \mathbb{N}$ we define a new metric $d_{n}$ on $X: \forall x, y \in X, d_{n}(x, y)=\max _{0 \leq i \leq n-1} d\left(T^{i} x, T^{i} y\right)$. Let $K \subseteq X$ and $\epsilon>0$. A subset $F \subseteq X$ is said to be $(n, \epsilon)$ span $K$ with respect to $T$ if for any $x \in K$, there exists some $y \in F$ with $d_{n}(x, y) \leq \epsilon$. A subset $E \subseteq K$ is said to be $(n, \epsilon)$ separated with respect to $T$ if $x, y \in E, x \neq y$ implies $d_{n}(x, y)>\epsilon$. Denote by $r_{n}(d, T, \epsilon, K)$ (resp. $\left.s_{n}(d, T, \epsilon, K)\right)$ the smallest (resp. largest) cardinality of any $(n, \epsilon)$-spanning subset 
(resp. $(n, \epsilon)$ separated subset) for $K$ with respect to $T$. Then following from some simple arguments, we have $r_{n}(d, T, \epsilon, K) \leq s_{n}(d, T, \epsilon, K) \leq r_{n}\left(d, T, \frac{\epsilon}{2}, K\right)$. Set

$r(d, T, \epsilon, K)=\limsup _{n \rightarrow+\infty} \frac{\log r_{n}(d, T, \epsilon, K)}{n}$ and $s(d, T, \epsilon, K)=\limsup _{n \rightarrow+\infty} \frac{\log s_{n}(d, T, \epsilon, K)}{n}$.

Obviously, $r(d, T, \epsilon, K)$ and $s(d, T, \epsilon, K)$ both increase when $\epsilon>0$ decreases to zero, and $r(d, T, \epsilon, K) \leq s(d, T, \epsilon, K) \leq r\left(d, T, \frac{\epsilon}{2}, K\right)$. Then define the limit

$$
\lim _{\epsilon \rightarrow 0+} r(d, T, \epsilon, K)=\lim _{\epsilon \rightarrow 0+} s(d, T, \epsilon, K)
$$

to be the topological entropy of $K$ with respect to $T$ (denoted by $h(d, T, K)$ ), and define the topological entropy of $(X, T)$ as $h(d, T)=\sup _{K} h(d, T, K)$ where the supremum is taken over all compact subsets of $X$, equivalently $h(d, T)=h(d, T, X)$. The following result is easy to check:

Lemma 2.1. Let $(X, T)$ be a TDS and d a metric on $X$.

1: Let $K_{1}, \cdots, K_{m} \subseteq X(m \in \mathbb{N})$. Then $r\left(d, T, \epsilon, \bigcup_{i=1}^{m} K_{i}\right)=\max _{1 \leq i \leq m}$ $r\left(d, T, \epsilon, K_{i}\right)$ for each $\epsilon>0$, and so $h\left(d, T, \bigcup_{i=1}^{m} K_{i}\right)=\max _{1 \leq i \leq m} h\left(d, T, K_{i}\right)$.

2: Bowen's definition of topological entropy depends only on the topology on $X$. Precisely, let $d^{\prime}$ be another metric on $X$ which is compatible with $d$. Then for any given $\epsilon_{1}>0$ there exist $0<\epsilon_{3}<\epsilon_{2}<\epsilon_{1}$ such that for each $K \subseteq X$ one has $r\left(d, T, \epsilon_{1}, K\right) \leq r\left(d^{\prime}, T, \epsilon_{2}, K\right) \leq r\left(d, T, \epsilon_{3}, K\right)$.

For a given TDS $(X, T)$ and $K \subseteq X$, Lemma 2.1 tells us that $h(d, T, K)$ is independent of the selection of the metric $d$, and so in the following we write $h(d, T, K)$ by $h(T, K)$, and $h(d, T)$ by $h(T)$ or $h(T, X)$. Lemma 2.1 stimulates the following

Definition 2.2. Let $(X, T)$ be a TDS.

(1) We say $x \in X$ is an entropy point if $h(T, K)>0$ for each closed neighborhood $K$ of $x$. Denote by $E_{p}(X, T)$ the set of all entropy points.

(2) We say $x \in X$ is a full entropy point if $h(T, K)=h(T)>0$ for each closed neighborhood $K$ of $x$. Denote by $E_{p}^{f}(X, T)$ the set of all full entropy points.

It is clear that if $(X, T)$ is a TDS with zero entropy, then $E_{p}^{f}(X, T)=E_{p}(X, T)=$ $\emptyset$. In general, $E_{p}^{f}(X, T) \subseteq E_{p}(X, T)$, and both $E_{p}(X, T)$ and $E_{p}^{f}(X, T)$ are closed. Moreover, let $(X, T)$ and $(Y, S)$ be two TDSs; by a factor map we mean a continuous surjective map $\pi:(X, T) \rightarrow(Y, S)$ with $S \pi=\pi T$. If in addition it is one to one, then we say $\pi$ is an isomorphism. We have

Proposition 2.3. Let $\pi:(X, T) \rightarrow(Y, S)$ be a factor map between TDSs. Then

1: $\pi\left(E_{p}^{f}(X, T)\right) \supseteq E_{p}^{f}(Y, S)$ when $h(T)=h(S)>0$.

2: $\pi\left(E_{p}(X, T)\right) \supseteq E_{p}(Y, S)$.

3: Moreover, if the factor map $\pi$ is open, then we have

(1) $\pi^{-1}\left(E_{p}^{f}(Y, S)\right) \subseteq E_{p}^{f}(X, T)$ when $h(T)=h(S)>0$.

(2) $\pi^{-1}\left(E_{p}(Y, S)\right) \subseteq E_{p}(X, T)$.

Proof. Say $d^{X}$ and $d^{Y}$ are metrics on TDSs $(X, T)$ and $(Y, S)$, respectively. 
Claim. If $\epsilon>0$, then there exists $\epsilon^{\prime}>0$ such that $r\left(d^{Y}, S, \epsilon, \pi(J)\right) \leq r\left(d^{X}, T, \epsilon^{\prime}, J\right)$ for any given subset $J \subseteq X$. Moreover, $h(S, \pi(J)) \leq h(T, J)$ when $J \subseteq X$.

Proof of the Claim. Assume $\epsilon>0$ and let $\epsilon^{\prime}>0$ such that $\epsilon>\epsilon^{\prime}$ and for $x_{1}^{\prime}, x_{2}^{\prime} \in X$, $d^{X}\left(x_{1}^{\prime}, x_{2}^{\prime}\right) \leq \epsilon^{\prime}$ implies $d^{Y}\left(\pi x_{1}^{\prime}, \pi x_{2}^{\prime}\right) \leq \epsilon$. Now let $E$ be an $\left(n, \epsilon^{\prime}\right)$-spanning set for $J$ with respect to $T$ with the smallest cardinality $r_{n}\left(d^{X}, T, \epsilon^{\prime}, J\right)$; then $\pi(E)$ is an $(n, \epsilon)$-spanning set for $\pi(J)$ with respect to $S$. Thus $r_{n}\left(d^{Y}, S, \epsilon, \pi(J)\right) \leq$ $r_{n}\left(d^{X}, T, \epsilon^{\prime}, J\right)$ which implies $r\left(d^{Y}, S, \epsilon, \pi(J)\right) \leq r\left(d^{X}, T, \epsilon^{\prime}, J\right)$. Letting $\epsilon \rightarrow 0+$ we have $h(S, \pi(J)) \leq h(T, J)$.

1. Assume $h(T)=h(S)>0$. Let $y_{1} \in E_{p}^{f}(Y, S)$ and for each $n \geq 2$ let $K_{n}$ be a closed neighborhood of $y_{1}$ with diameter at most $\frac{1}{n}$. Then by the Claim

$$
h\left(T, \pi^{-1} K_{n}\right) \geq h\left(S, K_{n}\right)=h(S)=h(T)>0 .
$$

Cover $\pi^{-1} K_{n}$ by closed balls with diameter at most $\frac{1}{n}$. Then there is such a closed ball $B_{n}$ with $h\left(T, B_{n}\right)=h\left(T, \pi^{-1} K_{n}\right)$ by Lemma 2.1. Let $x_{1}$ be a limit point of the sequence of balls $\left\{B_{n}: n \in \mathbb{N}\right\}$. Then $\pi\left(x_{1}\right)=y_{1}$ and it is easy to see $x_{1} \in E_{p}^{f}(X, T)$. That is, $\pi\left(E_{p}^{f}(X, T)\right) \supseteq E_{p}^{f}(Y, S)$.

2. Following the proof of 1 we get 2 .

3. We just show $\pi^{-1}\left(E_{p}^{f}(Y, S)\right) \subseteq E_{p}^{f}(X, T)$, and the other case follows similarly.

Assume that $h(T)=h(S)>0$. Let $y_{2} \in E_{p}^{f}(Y, S)$ and $x_{2} \in X$ with $\pi\left(x_{2}\right)=y_{2}$. For each $n \in \mathbb{N}$, let $K_{n}$ be a closed neighborhood of $x_{2}$ with diameter at most $\frac{1}{n}$ such that the diameter of $\pi\left(K_{n}\right)$ is also at most $\frac{1}{n}$. Since $\pi$ is open, $\pi\left(K_{n}\right)$ is also a closed neighborhood of $y_{2}$, and so $h\left(S, \pi\left(K_{n}\right)\right)=h(S)$ as $y_{2} \in E_{p}^{f}(Y, S)$. Then

$$
h(T) \geq h\left(T, K_{n}\right) \geq h\left(S, \pi\left(K_{n}\right)\right) \text { (by the Claim) }=h(S)=h(T)>0 .
$$

That is, $x_{2} \in E_{p}^{f}(X, T)$, which implies $\pi^{-1}\left(E_{p}^{f}(Y, S)\right) \subseteq E_{p}^{f}(X, T)$.

The next proposition says that in a positive entropy system $E_{p}^{f}(X, T)$ is not empty.

Proposition 2.4. Let $(X, T)$ be a TDS. Then

1: $E_{p}^{f}(X, T) \neq \emptyset$ when $h(T)>0$.

2: Both $E_{p}(X, T)$ and $E_{p}^{f}(X, T)$ are $T$-invariant.

Consequently, if $T$ is minimal and $h(T)>0$, then $E_{p}^{f}(X, T)=E_{p}(X, T)=X$.

Proof. Since $E_{p}(X, T)$ and $E_{p}^{f}(X, T)$ are closed, we only need to show parts 1 and 2.

1. Cover $X$ by finitely many closed balls $B_{1}^{1}, \cdots, B_{i_{1}}^{1}$ with diameter at most 1 . By Lemma 2.1. as $h(T)=h(T, X)=\max _{j} h\left(T, B_{j}^{1}\right)$, there is $j_{1}$ with $h(T)=h\left(T, B_{j_{1}}^{1}\right)$. Cover $B_{j_{1}}^{1}$ by finitely many closed balls $B_{1}^{2}, \cdots, B_{i_{2}}^{2}$ of $B_{j_{1}}^{1}$ with diameter at most $\frac{1}{2}$. By the same reasoning, there is $j_{2}$ with $h(T)=h\left(T, B_{j_{2}}^{2}\right)$. By induction, for each $n \geq 2$, there is a closed ball $B_{j_{n}}^{n}$ of $B_{j_{n-1}}^{n-1}$ with diameter at most $\frac{1}{n}$ such that $h(T)=h\left(T, B_{j_{n}}^{n}\right)$.

Set $\{x\}=\bigcap_{n \in \mathbb{N}} B_{j_{n}}^{n}$. It is clear that $x$ is a full entropy point when $h(T)>0$.

2. Since $T:(X, T) \rightarrow(X, T)$ and $T^{-1}:(X, T) \rightarrow(X, T)$ are isomorphisms between TDSs, applying Proposition 2.3 to $T$ and $T^{-1}$ we obtain that $T\left(E_{p}(X, T)\right)=$ $E_{p}(X, T)$ and $T\left(E_{p}^{f}(X, T)\right)=E_{p}^{f}(X, T)$. 
Moreover, using the same method as in the proof of Proposition 2.4 we have

Proposition 2.5. Let $(X, T)$ be a TDS and $K \subseteq X$ a closed subset.

1: If $h(T, K)>0$, then $K \cap E_{p}(X, T) \neq \emptyset$.

2: If $h(T, K)=h(T)>0$, then $K \cap E_{p}^{f}(X, T) \neq \emptyset$.

\section{Structure of the Set of Entropy Points}

Based on the theories and results established in [2], [5], 15] and [19], in this section we study the structure of the set of entropy points. It is proved that the support of any ergodic measure with positive entropy is contained in the set of entropy points, and the topological entropy of a TDS equals the topological entropy over the set of entropy points whereas it doesn't hold for the set of full entropy points, as $E_{p}^{f}(X, T)$ could be a singleton for some positive entropy TDS.

Let $(X, T)$ be a TDS. Denote by $\mathcal{M}(X, T)$ and $\mathcal{M}^{e}(X, T)$ respectively the set of all invariant probability measures and all ergodic invariant probability measures on $\left(X, \mathcal{B}_{X}, T\right)$, where $\mathcal{B}_{X}$ is the Borel $\sigma$-algebra of $X$. Let $\mu \in \mathcal{M}(X, T)$. For a given finite measurable partition $\mathbb{P}$, write $\mathbb{P}_{0}^{n-1}=\bigvee_{i=0}^{n-1} T^{-i} \mathbb{P}(\forall n \in \mathbb{N})$ and $\mathbb{P}^{-}=\bigvee_{i \geq 1} T^{-i} \mathbb{P}$. The entropy of $\mu$ for $\mathbb{P}$ is defined as

$$
h_{\mu}(T, \mathbb{P})=\lim _{n \rightarrow+\infty} \frac{1}{n} H_{\mu}\left(\mathbb{P}_{0}^{n-1}\right)=H_{\mu}\left(\mathbb{P} \mid \mathbb{P}^{-}\right) .
$$

The entropy of $\mu$ is given by $h_{\mu}(T)=\sup h_{\mu}(T, \mathbb{P})$, where $\mathbb{P}$ ranges over all finite measurable partitions of $\left(X, \mathcal{B}_{X}, T\right)$. The variational principle states that

$$
h(T)=\sup _{\mu \in \mathcal{M}(X, T)} h_{\mu}(T)=\sup _{\mu \in \mathcal{M}^{e}(X, T)} h_{\mu}(T) .
$$

The notion of entropy pair introduced in [2, 5] is generalized to entropy tuple in [15] both in topological and measure-theoretical settings. For each $n \geq 2$ and $\mu \in \mathcal{M}(X, T)$, denote by $E_{n}(X, T)$ the set of entropy $n$-tuples, and by $E_{n}^{\mu}(X, T)$ the set of entropy $n$-tuples for $\mu$.

Let $(X, T)$ be a TDS, $\mu \in \mathcal{M}(X, T)$ and measure-theoretical dynamical system (MDS, for short) $(Y, \mathcal{D}, \nu, S)$ the Pinsker factor of $(X, \mathcal{B}, \mu, T)$, where $\mathcal{B}$ is the completion of $\mathcal{B}_{X}$ under $\mu$. For each $n \geq 2$, define an invariant probability measure $\lambda_{n}(\mu)$ on $\left(X^{(n)}, \mathcal{B}^{(n)}, T^{(n)}\right)$ by letting

$$
\lambda_{n}(\mu)\left(\prod_{i=1}^{n} A_{i}\right)=\int_{X} \prod_{i=1}^{n} \mathbb{E}\left(1_{A_{i}} \mid \mathcal{D}\right) d \mu
$$

where $X^{(n)}=X \times \cdots \times X(n$ times $), \mathcal{B}^{(n)}=\mathcal{B} \times \cdots \times \mathcal{B}(n$ times $), T^{(n)}=T \times \cdots \times T$ ( $n$ times $), A_{i} \in \mathcal{B}$ and $\mathbb{E}\left(1_{A_{i}} \mid \mathcal{D}\right)$ is the conditional expectation of $1_{A_{i}}$ with respect to $\mathcal{D}(1 \leq i \leq n)$. Let $\mu=\int_{Y} \mu_{y} d \nu(y)$ be the disintegration of $\mu$ over $\nu$; it's wellknown that $\lambda_{n}(\mu)=\int_{Y} \mu_{y}^{(n)} d \nu(y)$ where $\mu_{y}^{(n)}=\mu_{y} \times \cdots \times \mu_{y}$ ( $n$ times $)$. Denote by $\Delta_{n}(X)=\left\{\left(x_{i}\right)_{1}^{n} \in X^{(n)}: x_{1}=\cdots=x_{n}\right\}$, the $n$-th diagonal of $X$. Then $E_{n}(X, T)$ (resp. $\left.E_{n}^{\mu}(X, T)\right)$ is $T^{(n)}$-invariant and $E_{n}(X, T) \cup \Delta_{n}(X)\left(\operatorname{resp} . E_{n}^{\mu}(X, T) \cup \Delta_{n}(X)\right)$ is a closed subset of $X^{(n)}$. Moreover, one has (see Theorems 4.4, 4.9, 6.1 and 6.4 in 15$]$ :

Proposition 3.1. Let $(X, T)$ be a TDS and $\mu \in \mathcal{M}(X, T)$. Then

1: $E_{n}(X, T) \supseteq E_{n}^{\mu}(X, T)=\operatorname{Supp}\left(\lambda_{n}(\mu)\right) \backslash \Delta_{n}(X)$ for each $n \geq 2$.

2: There is $\mu_{0} \in \mathcal{M}(X, T)$ with $E_{n}^{\mu_{0}}(X, T)=E_{n}(X, T)$ for each $n \geq 2$. 
Proposition 3.2. Let $(X, T)$ be a TDS and $\mu \in \mathcal{M}(X, T)$. Say $\mu=\int_{\Omega} \mu_{\omega} d \eta(\omega)$ is the ergodic decomposition of $\mu$. Then we have

1: $E_{n}^{\mu_{\omega}}(X, T) \subseteq E_{n}^{\mu}(X, T)$ for each $n \geq 2$ and $\eta$-a.e. $\omega \in \Omega$.

2: If $\left(x_{i}\right)_{1}^{n} \in E_{n}^{\mu}(X, T)$, then $\eta\left(\left\{\omega \in \Omega \mid \prod_{i=1}^{n} V_{i} \cap E_{n}^{\mu_{\omega}}(X, T) \neq \emptyset\right\}\right)>0$ for any measurable neighborhood $V_{i}$ of $x_{i}(1 \leq i \leq n)$. Thus for an appropriate choice of $\Omega$

$$
\overline{\bigcup\left\{E_{n}^{\mu_{\omega}}(X, T): \omega \in \Omega\right\}} \backslash \Delta_{n}(X)=E_{n}^{\mu}(X, T) .
$$

Moreover, in [15] the authors proved (see Theorem 7.3 in [15])

Lemma 3.3. Let $(X, T)$ be a TDS with $\left(x_{i}\right)_{1}^{n} \notin \Delta_{n}(X)(n \geq 2)$. Then $\left(x_{i}\right)_{1}^{n} \in$ $E_{n}(X, T)$ iff (if and only if) for any given neighborhood $U_{i}$ of $x_{i}(1 \leq i \leq n)$, there exist some $b>0$ and $M_{b} \in \mathbb{N}$ such that for any $m \geq M_{b}$ we can find $J_{m} \subseteq\{1,2, \cdots, m\}$ satisfying $\left|J_{m}\right| \geq b m$ and $\bigcap_{j \in J_{m}} T^{-j} U_{s(j)} \neq \emptyset$ when $s \in$ $\{1,2, \cdots, n\}^{J_{m}}$.

Now we are ready to show

Theorem 3.4. Let $(X, T)$ be a TDS and $\left(x_{i}\right)_{1}^{n} \in E_{n}(X, T)(n \geq 2)$. Then $\left\{x_{1}, \cdots, x_{n}\right\} \subseteq E_{p}(X, T)$.

Proof. We only need to prove $x_{1} \in E_{p}(X, T)$. Let $d$ be a metric on $X$.

Without loss of generality we assume $x_{i} \neq x_{j}$ if $i \neq j$. Let $U_{i}$ be any closed neighborhood of $x_{i}(1 \leq i \leq n)$ with $U_{i} \cap U_{j}=\emptyset$ if $i \neq j$. Then $\epsilon=\min _{1 \leq i<j \leq n} d\left(U_{i}, U_{j}\right)$ $>0$. Let $b$ and $M_{b}$ be the constants constructed in Lemma 3.3. Then for any $m \geq M_{b}$ there exists $J_{m} \subseteq\{1,2, \cdots, m\}$ such that $\left|J_{m}\right| \geq b m$ and when $s \in$ $\{1,2, \cdots, n\}^{J_{m}}$,

$$
\bigcap_{j \in J_{m}} T^{-j} U_{s(j)} \neq \emptyset \text {, say } x_{s} \in \bigcap_{j \in J_{m}} T^{-j} U_{s(j)} \text {, and so } T^{j} x_{s} \in U_{s(j)}\left(\forall j \in J_{m}\right) .
$$

Set $j_{m}$ to be the minimal element in $J_{m}$ and

$$
E=\left\{T^{j_{m}} x_{s}: s \in\{1,2, \cdots, n\}^{J_{m}} \text { with } s\left(j_{m}\right)=1\right\} \subseteq U_{1} .
$$

Obviously $|E|=n^{\left|J_{m}\right|-1} \geq n^{b m-1}$. Note that for $s_{1}, s_{2} \in\{1,2, \cdots, n\}^{J_{m}}: s_{1} \neq s_{2}$ and $s_{1}\left(j_{m}\right)=s_{2}\left(j_{m}\right)=1$, say $k_{m} \in J_{m}$ with $s_{1}\left(k_{m}\right) \neq s_{2}\left(k_{m}\right)$, then

$$
d_{m}\left(T^{j_{m}} x_{s_{1}}, T^{j_{m}} x_{s_{2}}\right) \geq d\left(T^{k_{m}} x_{s_{1}}, T^{k_{m}} x_{s_{2}}\right) \geq d\left(U_{s_{1}\left(k_{m}\right)}, U_{s_{2}\left(k_{m}\right)}\right) \geq \epsilon .
$$

This means that $E$ forms an $\left(m, \frac{\epsilon}{2}\right)$ separated subset of $U_{1}$ with respect to $T$, which implies $s_{m}\left(d, T, \frac{\epsilon}{2}, U_{1}\right) \geq n^{b m-1}$. Thus $h\left(d, T, U_{1}\right) \geq s\left(d, T, \frac{\epsilon}{2}, U_{1}\right) \geq b \log n>0$, and hence $x_{1} \in E_{p}(X, T)$. This ends the proof.

Let $(X, T)$ be a TDS and let $n \geq 2$. Denote by $p_{n}: X^{(n)} \rightarrow X$ the projection to the first coordinate. Then we have $p_{n}\left(E_{n}(X, T)\right) \subseteq E_{p}(X, T)$ (by Theorem 3.4) and so $\overline{p_{n}\left(E_{n}(X, T)\right)} \subseteq E_{p}(X, T)$. Moreover

Theorem 3.5. Let $(X, T)$ be a TDS. Then

1: If $\mu \in \mathcal{M}^{e}(X, T)$ satisfies $h_{\mu}(T)>0$, then $\operatorname{Supp}(\mu) \subseteq E_{p}(X, T)$.

2: $h\left(T, E_{p}(X, T)\right)=h(T)$. 
Proof. 1. Let $\mu=\int_{Y} \mu_{y} d \nu(y)$ be the disintegration of $\mu$ over its Pinsker factor. Since

$$
1=\lambda_{2}(\mu)\left(\operatorname{Supp}\left(\lambda_{2}(\mu)\right)\right)=\int_{Y} \mu_{y} \times \mu_{y}\left(\operatorname{Supp}\left(\lambda_{2}(\mu)\right)\right) d \nu(y),
$$

there exists some measurable $Y_{2} \subseteq Y$ such that $\nu\left(Y_{2}\right)=1$ and $\mu_{y} \times \mu_{y}\left(\operatorname{Supp}\left(\lambda_{2}(\mu)\right)\right)$ $=1$ for each $y \in Y_{2}$. Then by Proposition 3.1, when $y \in Y_{2}$

$$
\operatorname{Supp}\left(\mu_{y}\right) \times \operatorname{Supp}\left(\mu_{y}\right) \subseteq \operatorname{Supp}\left(\lambda_{2}(\mu)\right) \subseteq E_{2}(X, T) \cup \Delta_{2}(X),
$$

as $\operatorname{Supp}\left(\mu_{y} \times \mu_{y}\right)=\operatorname{Supp}\left(\mu_{y}\right) \times \operatorname{Supp}\left(\mu_{y}\right)$. Since $\mu \in \mathcal{M}^{e}(X, T)$ and $h_{\mu}(T)>0$, it's well-known that for $\nu$-a.e. $y \in Y, \operatorname{Supp}\left(\mu_{y}\right)$ is a compact subset without isolated points (see for example Lemma 4.1 in [22]). Now applying Theorem 3.4 to (3.3) one has, for $\nu$-a.e. $y \in Y, \operatorname{Supp}\left(\mu_{y}\right) \subseteq E_{p}(X, T)$. This implies $\operatorname{Supp}(\mu) \subseteq E_{p}(X, T)$.

2. Using 1 it is a direct application of the variational principle.

Similar to Bowen's definition of topological entropy, Katok 19 has given an analogous description of measure-theoretical entropy and proved the following result.

Lemma 3.6. Let $(X, T)$ be a TDS, $d$ a metric on $X$ and $\mu \in \mathcal{M}^{e}(X, T)$. For $\epsilon>0$ and $1>\delta>0$, denote by $r_{n}(d, T, \epsilon, \delta, \mu)$ the minimum number of $\epsilon$-balls in the $d_{n}$ metric whose union has $\mu$-measure at least $1-\delta$. Then for each $1>\delta>0$ we have

$$
h_{\mu}(T)=\lim _{\epsilon \rightarrow 0+} \limsup _{n \rightarrow \infty} \frac{1}{n} \log r_{n}(d, T, \epsilon, \delta, \mu) .
$$

With the help of Lemma 3.6 we have

Theorem 3.7. Let $(X, T)$ be a TDS, $d$ a metric on $X$ and $\mu \in \mathcal{M}^{e}(X, T)$. Then

$$
\lim _{\epsilon \rightarrow 0+} \inf \left\{s(d, T, \epsilon, K): K \in \mathcal{B}_{X} \text { with } \mu(K)>0\right\} \geq h_{\mu}(T) .
$$

In particular, for any $K \in \mathcal{B}_{X}, h(d, T, K) \geq h_{\mu}(T)$ if $\mu(K)>0$.

Proof. For the proof it is sufficient to show (3.5). Let $1>\delta>0$ be a given constant.

Let $\epsilon>0$ and $K \in \mathcal{B}_{X}$ satisfy $\mu(K)>0$. Since $\mu \in \mathcal{M}^{e}(X, T)$, there exists $m(K) \in \mathbb{Z}_{+}$such that $\mu\left(\bigcup_{i=0}^{m(K)} T^{i} K\right) \geq 1-\delta$.

Claim: For each $m \in \mathbb{Z}_{+}, s(d, T, \epsilon, K)=s\left(d, T, \epsilon, \bigcup_{i=0}^{m} T^{i} K\right)$.

Proof of the Claim. For each $n \in \mathbb{N}$, let $E \subseteq \bigcup_{i=0}^{m} T^{i} K$ be any $(n, \epsilon)$ separated subset of $\bigcup_{i=0}^{m} T^{i} K$ with respect to $T$. Then there exists some $i_{0}(n): 0 \leq i_{0}(n) \leq m$ such that $\left|E \cap T^{i_{0}(n)} K\right| \geq \frac{|E|}{m+1}$. Note that $T^{-i_{0}(n)} E \cap K$ is an $\left(n+i_{0}(n), \epsilon\right)$ separated (hence $(n+m, \epsilon)$ separated) subset of $K$ with respect to $T$. One has

$$
s_{n+m}(d, T, \epsilon, K) \geq \frac{1}{m+1} s_{n}\left(d, T, \epsilon, \bigcup_{i=0}^{m} T^{i} K\right) \geq \frac{1}{m+1} s_{n}(d, T, \epsilon, K),
$$

which implies $s(d, T, \epsilon, K)=s\left(d, T, \epsilon, \bigcup_{i=0}^{m} T^{i} K\right)$. This ends the proof of the Claim. 
Since $r_{n}(d, T, \epsilon, K) \leq s_{n}(d, T, \epsilon, K)$ for each $n \in \mathbb{N}$, by the Claim we have

$$
\begin{aligned}
& \inf \left\{s(d, T, \epsilon, K): K \in \mathcal{B}_{X} \text { with } \mu(K)>0\right\} \\
= & \inf \left\{s\left(d, T, \epsilon, \bigcup_{i=0}^{m(K)} T^{i} K\right): K \in \mathcal{B}_{X} \text { with } \mu(K)>0\right\} \\
\geq & \inf \left\{\limsup _{n \rightarrow \infty} \frac{1}{n} \log r_{n}\left(d, T, \epsilon, \bigcup_{i=0}^{m(K)} T^{i} K\right): K \in \mathcal{B}_{X} \text { with } \mu(K)>0\right\} \\
\geq & \limsup _{n \rightarrow \infty} \frac{1}{n} \inf \left\{\log r_{n}\left(d, T, \epsilon, \bigcup_{i=0}^{m(K)} T^{i} K\right): K \in \mathcal{B}_{X} \text { with } \mu(K)>0\right\} \\
\geq & \limsup _{n \rightarrow \infty} \frac{1}{n} \log r_{n}(d, T, \epsilon, \delta, \mu) \text { (in the notation of Lemma 3.6). }
\end{aligned}
$$

Letting $\epsilon \rightarrow 0+$, the inequality (3.5) follows directly from the above discussion and Lemma 3.6. This ends the proof.

As an easy application of Theorem 3.7, we have

Corollary 3.8. Let $(X, T)$ be a TDS with positive entropy. Then we have

$$
\overline{\bigcup\left\{\operatorname{Supp}(\mu): \mu \in \mathcal{M}^{e}(X, T), h_{\mu}(T)=h(T)\right\}} \subseteq E_{p}^{f}(X, T) .
$$

Corollary 3.8 says that if there is an ergodic measure with maximal entropy, then $h\left(T, E_{p}^{f}(X, T)\right)=h(T)$. Now we describe a TDS which has no ergodic measure with maximal entropy such that $E_{p}^{f}(X, T)$ is a singleton.

Example 3.9. For any given $b>0$ (including $b=\infty)$ there exists a TDS $(X, T)$ such that $h(T)=b$ and $E_{p}^{f}(X, T)$ is a singleton.

Proof. Let $b>0$ be fixed. Clearly, there exist $b>b_{n}>0$ with $b_{n} \rightarrow b$ and a sequence $\left\{\left(X_{n}, T_{n}\right)\right\}_{n \in \mathbb{N}}$ of TDSs satisfying $h\left(T_{n}\right)=b_{n}(\forall n \in \mathbb{N})$ and $X_{n} \subset \mathbb{R}^{2}$. Let $B_{n}$ be a sequence of disjoint closed balls in $\mathbb{R}^{2}$ with $\operatorname{diam}\left(B_{n}\right) \rightarrow 0,(0,0) \notin B_{n}$ and $B_{n} \rightarrow(0,0)$. Embed $X_{n}$ into $B_{n}$ for each $n$ and put $X=\bigcup_{n \in \mathbb{N}} X_{n} \cup\{(0,0)\}$. Define $T: X \rightarrow X$ such that $\left.T\right|_{X_{n}}=T_{n}$ and $(0,0)$ is a fixed point. Then $(X, T)$ is a TDS and $h(T)=b$.

It is clear that there is no ergodic measure with maximal entropy for $(X, T)$. Moreover, by definition and Proposition 2.4. $\emptyset \neq E_{p}^{f}(X, T) \subseteq X \backslash \bigcup_{n \in \mathbb{N}} X_{n}$; then $(0,0)$ is the unique full entropy point.

\section{Entropy FUnCtion}

In this section, as a tool for studying uniform entropy point appearing in the next section, we introduce the concept of entropy function and discuss its fundamental properties.

Let $(X, T)$ be a TDS and $d$ a metric on $X$. For each $\epsilon>0$ and $x \in X$, define

$$
h_{d}(x, \epsilon)=\inf \{r(d, T, \epsilon, K): K \text { is a closed neighborhood of } x\} .
$$


Obviously, $h_{d}(x, \epsilon)$ increases as $\epsilon$ decreases to zero. Put $h_{d}(x)=\lim _{\epsilon \rightarrow 0+} h_{d}(x, \epsilon) \leq$ $h(T)$. By Lemma 2.1, it's not hard to see that the value of $h_{d}(x)$ depends only on the topology of $X$. Thus in the sequel sometimes we will write $h_{d}(x)$ as $h(x)$.

Definition 4.1. Let $(X, T)$ be a TDS. The function $h: X \rightarrow[0, h(T)], x \mapsto h(x)$ is said to be the entropy function of $(X, T)$.

Since $r(d, T, \epsilon, K) \leq s(d, T, \epsilon, K) \leq r\left(d, T, \frac{\epsilon}{2}, K\right)$, we have

$$
h(x)=\lim _{\epsilon \rightarrow 0+} \inf \{s(d, T, \epsilon, K): K \text { is a closed neighborhood of } x\} .
$$

Then using the language of entropy function, Theorem 3.7 tells us that

Theorem 4.2. Let $(X, T)$ be a TDS, $h$ its entropy function and $x \in X$. Then

$$
h(x) \geq \sup \left\{h_{\mu}(T): \mu \in \mathcal{M}^{e}(X, T), x \in \operatorname{Supp}(\mu)\right\} .
$$

In particular, if $(X, T)$ is minimal, then $h \equiv h(T)$ is a constant function.

The following is the lift-up property of entropy function.

Proposition 4.3. Let $\pi:(X, T) \rightarrow(Y, S)$ be a factor map between TDSs. If $h^{X}$ and $h^{Y}$ are the entropy functions of $(X, T)$ and $(Y, S)$, respectively, then

1: For each $y_{1} \in Y, \sup _{x \in \pi^{-1}\left(y_{1}\right)} h^{X}(x) \geq h^{Y}\left(y_{1}\right)$.

2: Moreover, if $\pi$ is open, then $h^{X}(x) \geq h^{Y}\left(y_{2}\right)$ for all $y_{2} \in Y$ and $x \in$ $\pi^{-1}\left(y_{2}\right)$.

Proof. Say $d^{X}$ and $d^{Y}$ are metrics on TDSs $(X, T)$ and $(Y, S)$, respectively. Let $\epsilon>$ 0 ; then by the Claim of Proposition 2.3, there exists $\epsilon^{\prime}>0$ such that $r\left(d^{Y}, S, \epsilon, \pi(J)\right)$ $\leq r\left(d^{X}, T, \epsilon^{\prime}, J\right)$ if $J \subseteq X$. In particular, $r\left(d^{Y}, S, \epsilon, K\right) \leq r\left(d^{X}, T, \epsilon^{\prime}, \pi^{-1}(K)\right)$ if $K \subseteq Y$.

1. $\forall n \in \mathbb{N}$, let $K_{n}$ be a closed neighborhood of $y_{1}$ with diameter at most $\frac{1}{n}$. By Lemma 2.1, there exists a closed $B_{n} \subseteq \pi^{-1}\left(K_{n}\right)$ with diameter at most $\frac{1}{n}$ such that

$$
r\left(d^{X}, T, \epsilon^{\prime}, B_{n}\right)=r\left(d^{X}, T, \epsilon^{\prime}, \pi^{-1}\left(K_{n}\right)\right) \geq r\left(d^{Y}, S, \epsilon, K_{n}\right) \geq h_{d^{Y}}^{Y}\left(y_{1}, \epsilon\right) .
$$

Let $x_{1}$ be a limit point of the sequence of closed subsets $\left\{B_{n}: n \in \mathbb{N}\right\}$. Obviously, $\pi\left(x_{1}\right)=y_{1}$ and $h_{d^{X}}^{X}\left(x_{1}, \epsilon^{\prime}\right) \geq h_{d^{Y}}^{Y}\left(y_{1}, \epsilon\right)$. This means $\sup _{x \in \pi^{-1}\left(y_{1}\right)} h^{X}(x) \geq$ $h_{d^{Y}}^{Y}\left(y_{1}, \epsilon\right)$. Then the conclusion follows by letting $\epsilon \rightarrow 0+$.

2. Let $x_{2} \in \pi^{-1}\left(y_{2}\right)$ : fixed. $\forall n \in \mathbb{N}$, let $K_{n}$ be a closed neighborhood of $x_{2}$ with diameter at most $\frac{1}{n}$ such that the diameter of $\pi\left(K_{n}\right)$ is less than $\frac{1}{n}$. As $\pi$ is open, $\pi\left(K_{n}\right)$ is a closed neighborhood of $y_{2}$ and

$$
r\left(d^{X}, T, \epsilon^{\prime}, K_{n}\right) \geq r\left(d^{Y}, S, \epsilon, \pi\left(K_{n}\right)\right) \geq h_{d^{Y}}^{Y}\left(y_{2}, \epsilon\right) .
$$

Then $h^{X}\left(x_{2}\right) \geq h_{d^{X}}^{X}\left(x_{2}, \epsilon^{\prime}\right) \geq h_{d^{Y}}^{Y}\left(y_{2}, \epsilon\right)$ and so $h^{X}\left(x_{2}\right) \geq h^{Y}\left(y_{2}\right)$.

Let $f$ be an extended real-valued function defined on a compact metric space $Z$. $f$ is called upper semi-continuous (u.s.c., for short) if for each $r \in \mathbb{R}$ the subset $f^{-1}([-\infty, r))$ is open, equivalently, $\limsup _{z^{\prime} \rightarrow z} f\left(z^{\prime}\right) \leq f(z)$ for each $z \in Z$. Obviously, each u.s.c. function is Borel measurable. As immediate consequences of the definition, we have that the infimum of any family of u.s.c. functions is u.s.c., and both the sum and supremum of finitely many u.s.c. functions are u.s.c. 
Proposition 4.4. Let $(X, T)$ be a TDS, $d$ a metric on $X$ and $h$ its entropy function. Then

1: $h$ is $T$-invariant.

2: For each $\epsilon>0, h_{d}(\bullet, \epsilon)$ is u.s.c. on $X$ and so $h$ is Borel measurable.

3: If $K \subseteq X$ is closed, then $\sup _{x \in K} h(x) \geq h(T, K)$. In particular, $\sup _{x \in X} h(x)=$ $h(T)$.

Proof. 1. As $T:(X, T) \rightarrow(X, T)$ and $T^{-1}:(X, T) \rightarrow(X, T)$ are isomorphisms between TDSs, applying Proposition 4.3 to $T$ and $T^{-1}$ we obtain the conclusion.

2. Let $\epsilon>0$ be given. For each $r \in \mathbb{R}$, if $h_{d}\left(x_{0}, \epsilon\right)<r$, then $r(d, T, \epsilon, K)<r$ for some open neighborhood $K$ of $x_{0}$, which implies that $h_{d}(x, \epsilon)<r$ for each $x \in K$, that is, the function $h_{d}(\bullet, \epsilon)$ is u.s.c. on $X$. Moreover, $h$, as the limit function of a sequence of u.s.c. functions $\left\{h_{d}\left(\bullet, \frac{1}{n}\right)\right\}$, is Borel measurable on $X$.

3. It suffices to consider the case of $h(T, K)>0$. Let $\epsilon>0$ be fixed. Cover $K$ by finitely many closed balls $B_{1}^{1}, \cdots, B_{i_{1}}^{1}$ with diameter at most 1 . By Lemma 2.1. there is $j_{1}$ with $r(d, T, \epsilon, K)=r\left(d, T, \epsilon, B_{j_{1}}^{1} \cap K\right)$. Cover $B_{j_{1}}^{1}$ by finitely many closed balls $B_{1}^{2}, \cdots, B_{i_{2}}^{2}$ of $B_{j_{1}}^{1}$ with diameter at most $\frac{1}{2}$. By the same reasoning, there is $j_{2}$ with $r(d, T, \epsilon, K)=r\left(d, T, \epsilon, B_{j_{2}}^{2} \cap K\right)$. By induction, for each $n \geq 2$, there is a closed ball $B_{j_{n}}^{n}$ of $B_{j_{n-1}}^{n-1}$ with diameter at most $\frac{1}{n}$ satisfying $r(d, T, \epsilon, K)=$ $r\left(d, T, \epsilon, B_{j_{n}}^{n} \cap K\right)$.

Set $\left\{x_{0}\right\}=\bigcap_{n \in \mathbb{N}} B_{j_{n}}^{n}$; then $x_{0} \in K$, as $K$ is a closed subset. Let $K^{\prime}$ be any closed neighborhood of $x_{0}$. Then for each sufficiently large $n \in \mathbb{N}, B_{j_{n}}^{n} \cap K \subseteq K^{\prime}$ and so

$$
r\left(d, T, \epsilon, K^{\prime}\right) \geq r\left(d, T, \epsilon, B_{j_{n}}^{n} \cap K\right)=r(d, T, \epsilon, K),
$$

which implies $h\left(x_{0}\right) \geq h_{d}\left(x_{0}, \epsilon\right) \geq r(d, T, \epsilon, K)$. This means $\sup _{x \in K} h(x) \geq r(d, T, \epsilon, K)$. Then the conclusion follows by letting $\epsilon \rightarrow 0+$.

Then we have

Theorem 4.5. Let $(X, T)$ be a TDS and $h$ its entropy function. Then

1: For each $\mu \in \mathcal{M}^{e}(X, T)$, there is some $c$ with $h(T) \geq c \geq h_{\mu}(T)$ such that $h(x)=c$ for $\mu$-a.e. $x \in X$.

2: For each $\mu \in \mathcal{M}(X, T), \int_{X} h(x) d \mu(x) \geq h_{\mu}(T)$.

Proof. 1. Let $\mu \in \mathcal{M}^{e}(X, T)$. Since $h$ is $T$-invariant and $\sup _{x \in X} h(x)=h(T)$ (by Proposition 4.4), there exists some constant $c$ with $h(T) \geq c$ such that $h(x)=c$ for $\mu$-a.e. $x \in X$. Moreover, following from Theorem 4.2 one has $c \geq h_{\mu}(T)$.

2. Say $\mu=\int_{\Omega} \mu_{\omega} d \eta(\omega)$ is the ergodic decomposition. As $h$ is a non-negative Borel measurable function on $X$ (by Proposition 4.4), we have

$$
\int_{X} h(x) d \mu(x)=\int_{\Omega}\left\{\int_{X} h(x) d \mu_{\omega}(x)\right\} d \eta(\omega) .
$$

Now applying part 1 to (4.1) we obtain

$$
\int_{X} h(x) d \mu(x) \geq \int_{\Omega} h_{\mu_{\omega}}(T) d \eta(\omega)=h_{\mu}(T) \text { (by Theorem } 8.4 \text { of [21]). }
$$

This completes the proof. 
Remark 4.6. In [8, the authors considered a local version of the characterization of entropy, and obtained a theorem on local entropy, which can be viewed as a topological version of the well-known Shannon-MacMillan-Breiman Theorem (see for example [12]), whereas, it is just an almost everywhere definition for any given invariant measure over $(X, T)$. Precisely, if $\mu \in \mathcal{M}(X, T)$, then for $\mu$-a.e. $x \in X$

$$
\lim _{\epsilon \rightarrow 0+} \liminf _{n \rightarrow+\infty}-\frac{1}{n} \log \mu\left(B_{n}(x, \epsilon)\right)=\lim _{\epsilon \rightarrow 0+} \limsup _{n \rightarrow+\infty}-\frac{1}{n} \log \mu\left(B_{n}(x, \epsilon)\right),
$$

which is denoted by $h_{\mu}(x)$, where $B_{n}(x, \epsilon)$ denotes the open $d_{n}$-ball of $X$ with center $x$ and radius $\epsilon$. Moreover, $h_{\mu}(x)$ is $T$-invariant and $\int_{X} h_{\mu}(x) d \mu(x)=h_{\mu}(T)$. In particular, if $\mu \in \mathcal{M}^{e}(X, T)$, then $h_{\mu}(x)=h_{\mu}(T)$ for $\mu$-a.e. $x \in X$. Now using Theorem 4.5 we have that if $\mu \in \mathcal{M}^{e}(X, T)$, then $h_{\mu}(x)=h_{\mu}(T) \leq h(x)$ for $\mu$-a.e. $x \in X$.

\section{UNIFORM ENTROPY POINT AND UNIFORM FULL ENTROPY POINT}

Starting from the definitions of uniform entropy point and uniform full entropy point, we discuss their basic properties and characterize them using entropy function introduced in the previous section. Applying Theorem 3.7 we know that the support of an ergodic measure with positive entropy is contained in the set of uniform entropy points. A consequence of these results is that for any TDS there exists a countable closed subset whose Bowen entropy is equal to the entropy of the original system.

Definition 5.1. Let $(X, T)$ be a TDS and $h$ its entropy function.

(1) We say $x \in X$ is a uniform entropy point if $h(x)>0$. Denote by $E_{u p}(X, T)$ the set of all uniform entropy points.

(2) We say $x \in X$ is a uniform full entropy point if $h(x)=h(T)>0$. Denote by $E_{u p}^{f}(X, T)$ the set of all uniform full entropy points.

These notions are related to the notions of entropy point and full entropy point in the following way.

Proposition 5.2. Let $(X, T)$ be a TDS. Then

1: $E_{u p}^{f}(X, T) \subseteq E_{u p}(X, T) \cap E_{p}^{f}(X, T), E_{u p}(X, T) \subseteq E_{p}(X, T)$.

2: If $K \subseteq X$ is a closed subset with $h(T, K)>0$, then $K \cap E_{u p}(X, T) \neq \emptyset$. In particular, $E_{u p}(X, T) \neq \emptyset$ when $h(T)>0$.

3: $E_{u p}(X, T)$ and $E_{u p}^{f}(X, T)$ are both $T$-invariant.

4: $E_{u p}(X, T) \subseteq X$ is an $F_{\sigma}$ subset, and $E_{u p}^{f}(X, T) \subseteq X$ is an $F_{\sigma \delta}$ subset.

Proof. Part 1 follows directly from the definitions. Parts 2 and 3 are the easy consequences of Proposition 4.4. Now we prove part 4.

Without loss of generality we assume $h(T)>0$. Let $d$ be a metric on $X$. Then

$$
\begin{gathered}
E_{u p}(X, T)=\bigcup_{m \in \mathbb{N}} \bigcup_{n \in \mathbb{N}}\left\{x \in X: h_{d}\left(x, \frac{1}{n}\right) \geq \frac{1}{m}\right\} . \\
\text { If } h(T)<+\infty, E_{u p}^{f}(X, T)=\bigcap_{m \in \mathbb{N}} \bigcup_{n \in \mathbb{N}}\left\{x \in X: h_{d}\left(x, \frac{1}{n}\right) \geq h(T)-\frac{1}{m}\right\} . \\
\text { If } h(T)=+\infty, E_{u p}^{f}(X, T)=\bigcap_{m \in \mathbb{N}} \bigcup_{n \in \mathbb{N}}\left\{x \in X: h_{d}\left(x, \frac{1}{n}\right) \geq m\right\} .
\end{gathered}
$$


Note that for each $\epsilon>0$, the function $h_{d}(\bullet, \epsilon)$ is u.s.c. on $X$. Thus, it follows that $E_{u p}(X, T) \subseteq X$ is an $F_{\sigma}$ subset, and $E_{u p}^{f}(X, T) \subseteq X$ is an $F_{\sigma \delta}$ subset.

In general, for a TDS $(X, T)$ with positive entropy, we can't assert that $E_{u p}(X, T)$ is closed, or $E_{u p}^{f}(X, T)$ is closed or $E_{u p}^{f}(X, T) \neq \emptyset$, as the following example shows.

Example 5.3. We have the following examples:

1: For any given $b>0$ (including $b=\infty)$ there exists a TDS $(X, T)$ such that $h(T)=b, E_{u p}(X, T) \subseteq X$ is not closed and $E_{u p}^{f}(X, T)=\emptyset$.

2: For any given $b>0$ (including $b=\infty$ ) there exists a TDS $(X, T)$ such that $h(T)=b$ and $E_{u p}^{f}(X, T) \subseteq X$ is not closed.

Proof. 1. For a given $b>0$ (including $b=\infty)$, let $(X, T)$ and $\left\{\left(X_{n}, T_{n}\right)\right\}$ be the systems constructed in Example 3.9. Then $\forall n \in \mathbb{N}, E_{u p}(X, T) \supseteq E_{u p}\left(X_{n}, T_{n}\right) \neq \emptyset$ (by Proposition 5.2), which implies $(0,0)$ is contained in the closure of $E_{u p}(X, T)$. Now by definition it is easy to see that $h(0,0)=0$. Moreover, $(0,0) \notin E_{u p}(X, T)$ and $E_{u p}^{f}(X, T)=\emptyset$, where $h$ is the entropy function of $(X, T)$.

2. For a given $b>0$ (including $b=\infty$ ), let $(Y, S)$ be a TDS with a maximal ergodic measure $\mu$ with $h_{\mu}(S)=h(S)=b$. In the construction of Example 3.9 let $X_{n}=Y$ and $T_{n}=S$ for each $n \in \mathbb{N}$. Then $\forall n \in \mathbb{N}, h(T)=b$ and $E_{u p}^{f}(X, T) \supseteq$ $X_{n}$, and hence $(0,0)$ is in the closure of $E_{u p}^{f}(X, T)$. Similarly we have $(0,0) \notin$ $E_{u p}^{f}(X, T)$.

As a direct application of Theorem 4.2 we have

Corollary 5.4. Let $(X, T)$ be a TDS with positive entropy. Then

1: If $\mu \in \mathcal{M}^{e}(X, T)$ satisfies $h_{\mu}(T)>0$, then $\operatorname{Supp}(\mu) \subseteq E_{u p}(X, T)$.

2: If $\mu \in \mathcal{M}^{e}(X, T)$ satisfies $h_{\mu}(T)=h(T)>0$, then $\operatorname{Supp}(\mu) \subseteq E_{u p}^{f}(X, T)$.

3: If $(X, T)$ is minimal, then $E_{u p}(X, T)=E_{u p}^{f}(X, T)=X$.

4: $h\left(T, E_{\text {up }}(X, T)\right)=h(T)$.

Remark 5.5. Note that we let $(X, T)$ be an expansive TDS (that is, there exists $\delta>0$ such that $\sup _{n \in \mathbb{Z}} d\left(T^{n} x_{1}, T^{n} x_{2}\right)>\delta$ whenever $x_{1} \neq x_{2}$, where $d$ is a metric on $X)$. If $(X, T)$ has positive entropy, then it admits an ergodic measure with maximal entropy, so $E_{u p}^{f}(X, T) \neq \emptyset$ (by Corollary 5.4). The same holds for any asymptotically $h$-expansive TDS. As in [7] it was proved that each asymptotically $h$-expansive TDS admits a good extension to a symbolic TDS which is expansive. Moreover, it follows from the definition that for a full shift on finite symbols, each point is a uniform full entropy point.

For a factor map $\pi:(X, T) \rightarrow(Y, S)$ between TDSs, generally it doesn't hold that $\pi\left(E_{u p}^{f}(X, T)\right) \supseteq E_{u p}^{f}(Y, S)$. Nevertheless we have

Proposition 5.6. Let $\pi:(X, T) \rightarrow(Y, S)$ be a factor map between TDSs. Then

1: $\pi\left(E_{u p}(X, T)\right) \supseteq E_{u p}(Y, S)$.

2: Moreover, if the factor map $\pi$ is open, then we have

(1) $\pi^{-1}\left(E_{\text {up }}(Y, S)\right) \subseteq E_{\text {up }}(X, T)$.

(2) $\pi^{-1}\left(E_{u p}^{f}(Y, S)\right) \subseteq E_{u p}^{f}(X, T)$ when $h(T)=h(S)>0$.

Proof. Say $h^{X}$ and $h^{Y}$ are the entropy functions of $(X, T)$ and $(Y, S)$, respectively. 
1. $\forall y_{1} \in E_{u p}(Y, S): h^{Y}\left(y_{1}\right)>0$, fixed. By Proposition 4.3. there exists $x_{1} \in$ $\pi^{-1}\left(y_{1}\right)$ with $h^{X}\left(x_{1}\right)>0$ (equivalently, $x_{1} \in E_{u p}(X, T)$ ). That is, $\pi\left(E_{u p}(X, T)\right) \supseteq$ $E_{u p}(Y, S)$.

2. Since the proof is similar, we only show (2), the latter.

Let $x_{2} \in X$ with $y_{2} \doteq \pi\left(x_{2}\right) \in E_{u p}^{f}(Y, S)$ (equivalently, $h^{Y}\left(y_{2}\right)=h(S)>0$ ): fixed. Since the factor map $\pi$ is open and $h(T)=h(S)>0$, by Proposition 4.3 we have

$$
h(T) \geq h^{X}\left(x_{2}\right) \geq h^{Y}\left(y_{2}\right)=h(S)=h(T)>0 \Longrightarrow h^{X}\left(x_{2}\right)=h(T)>0,
$$

equivalently, $x_{2} \in E_{u p}^{f}(X, T)$. That is, $\pi^{-1}\left(E_{u p}^{f}(Y, S)\right) \subseteq E_{u p}^{f}(X, T)$.

In the remainder of this section, we study the entropy function using dynamical behavior of points in the system and apply these results to $E_{u p}(X, T)$ and $E_{u p}^{f}(X, T)$.

Theorem 5.7. Let $(X, T)$ be a TDS and $h$ its entropy function.

1: Suppose that $K$ is a countable closed subset of $X$ with a unique limit point $x_{0}$. Then $h\left(x_{0}\right) \geq h(T, K)$.

2: Suppose $x_{0} \in X$. Then there exists a countable closed subset $K \subseteq X$ such that $x_{0} \in K$ is its unique limit point in $X$ and $h(T, K)=h\left(x_{0}\right)$.

Proof. Say $d$ is a metric on $\operatorname{TDS}(X, T)$.

1. Let $\epsilon>0$ : fixed. By the assumption of $K$, for any closed neighborhood $W$ of $x_{0}, K \backslash W$ is finite which implies $r(d, T, \epsilon, W) \geq r(d, T, \epsilon, K)$. Then $h\left(x_{0}\right) \geq$ $h_{d}\left(x_{0}, \epsilon\right) \geq r(d, T, \epsilon, K)$. The conclusion follows by letting $\epsilon \rightarrow 0+$.

2 . Since the proof is similar, we only present a proof for the case when $h\left(x_{0}\right)<$ $+\infty$.

Set $K_{n}=\left\{x \in X: d\left(x, x_{0}\right) \leq \frac{1}{n}\right\}(\forall n \in \mathbb{N}) . \forall m \in \mathbb{N}$, choose $\epsilon_{m}>0$ such that

$$
h\left(x_{0}\right)-\frac{1}{m}<\inf _{n \in \mathbb{N}} s\left(d, T, \epsilon_{m}, K_{n}\right)=\inf _{n \in \mathbb{N}} \limsup _{k \rightarrow+\infty} \frac{\log s_{k}\left(d, T, \epsilon_{m}, K_{n}\right)}{k} .
$$

Then there exists a strictly increasing sequence $\left\{k_{n, m}\right\}_{n \in \mathbb{N}} \subseteq \mathbb{N}$ such that for all $n \in$ $\mathbb{N}, s_{k_{n, m}}\left(d, T, \epsilon_{m}, K_{n}\right) \geq e^{k_{n, m}\left(h\left(x_{0}\right)-\frac{1}{m}\right)}$. Say $E_{n, m} \subseteq K_{n}$ is a $\left(k_{n, m}, \epsilon_{m}\right)$ separated subset of $K_{n}$ with respect to $T$ with the largest cardinality $s_{k_{n, m}}\left(d, T, \epsilon_{m}, K_{n}\right)$.

Put $K=\bigcup_{m \geq 1} \bigcup_{n \geq m} E_{n, m} \cup\left\{x_{0}\right\}$. We prove that $K$ is just the subset we need.

If $V$ is a neighborhood of $x_{0}$, then $K_{n_{0}} \subseteq V$ for some $n_{0} \in \mathbb{N}$. Moreover

$$
K \backslash V \subseteq K \backslash K_{n_{0}} \subseteq \bigcup_{m=1}^{n_{0}-1} \bigcup_{n=m}^{n_{0}-1} E_{n, m}
$$

In particular, $K \backslash V$ is finite and so no other points except $x_{0}$ can be a limit point of $K$ in $X$. Thus $x_{0} \in K$ is the unique limit point of $K$ in $X$.

Now we show $h(T, K)=h\left(x_{0}\right)$. Let $m \in \mathbb{N}$ : fixed. When $n \geq m, E_{n, m} \subseteq K$ is $\left(k_{n, m}, \epsilon_{m}\right)$ separated with respect to $T$, so

$$
s_{k_{n, m}}\left(d, T, \epsilon_{m}, K\right) \geq\left|E_{n, m}\right|=s_{k_{n, m}}\left(d, T, \epsilon_{m}, K_{n}\right) \geq e^{k_{n, m}\left(h\left(x_{0}\right)-\frac{1}{m}\right)} .
$$


Thus

$$
\begin{aligned}
h(T, K) & \geq s\left(d, T, \epsilon_{m}, K\right)=\limsup _{k \rightarrow+\infty} \frac{\log s_{k}\left(d, T, \epsilon_{m}, K\right)}{k} \\
& \geq \limsup _{n \rightarrow+\infty} \frac{\log s_{k_{n, m}}\left(d, T, \epsilon_{m}, K\right)}{k_{n, m}} \\
& \geq \limsup _{n \rightarrow+\infty} \frac{\log e^{k_{n, m}\left(h\left(x_{0}\right)-\frac{1}{m}\right)}}{k_{n, m}}=h\left(x_{0}\right)-\frac{1}{m} .
\end{aligned}
$$

Since (5.1) holds for all $m \in \mathbb{N}$, letting $m \rightarrow+\infty$ we have $h(T, K) \geq h\left(x_{0}\right)$. Moreover, $h(T, K)=h\left(x_{0}\right)$ (using part 1). This completes the proof.

An immediately consequence of the above theorem is

Corollary 5.8. Let $(X, T)$ be a TDS with $x_{0} \in X$. Then

1: $x_{0} \in E_{u p}(X, T)$ iff there exists a countable closed subset $K \subseteq X$ such that $h(T, K)>0$ and $x_{0} \in K$ is its unique limit point in $X$.

2: $x_{0} \in E_{u p}^{f}(X, T)$ iff there exists a countable closed subset $K \subseteq X$ such that $h(T, K)=h(T)>0$ and $x_{0} \in K$ is its unique limit point in $X$.

Now, using Proposition 4.4 and Theorem 5.7 for a general TDS we could obtain the following unexpected result:

Theorem 5.9. Let $(X, T)$ be a TDS. Then there exists a countable closed subset $K \subseteq X$ such that $h(T, K)=h(T)$. Moreover, $K$ can be chosen such that the set of limit points of $K$ has at most one limit point, and $K$ has a unique limit point iff there is $x \in X$ with $h(x)=h(T)$.

Proof. Let $d$ be a metric on $X$ and $h$ its entropy function. For each $x \in X$ and any $\epsilon>0$, set $B_{x}(\epsilon)=\left\{x^{\prime} \in X: d\left(x, x^{\prime}\right)<\epsilon\right\}$.

As $X$ is compact, by Proposition 4.4 there exists $\left\{x_{n}\right\}_{n \in \mathbb{N}} \subseteq X$ such that

$$
\lim _{n \rightarrow+\infty} x_{n}=x_{0} \in X \text { and } \lim _{n \rightarrow+\infty} h\left(x_{n}\right)=h(T) .
$$

Let $\left\{r_{n}\right\}_{n \in \mathbb{N}}$ be any given sequence of positive real numbers with $r_{n} \rightarrow 0$ as $n \rightarrow \infty$. Using Theorem 5.7 for each $n \in \mathbb{N}$ we can select a countable closed subset $K_{n}$ such that $h\left(T, K_{n}\right)=h\left(x_{n}\right)$ and $x_{n}$ is its unique limit point in $X$. In particular, $K_{n} \backslash B_{x_{n}}\left(r_{n}\right)$ is a finite subset, so without loss of generality we assume $K_{n} \subseteq$ $B_{x_{n}}\left(r_{n}\right)$.

Set $K=\left\{x_{0}\right\} \cup \bigcup_{n \in \mathbb{N}} K_{n}$. Then $K$ is a countable closed subset of $X$ and the set of limit points of $K$ in $X$ is just $\left\{x_{0}\right\} \cup\left\{x_{n}: n \in \mathbb{N}\right\}$, as $x_{n} \rightarrow x_{0}$ and $r_{n} \rightarrow 0$ when $n \rightarrow \infty$. Obviously, $h(T, K) \geq h\left(T, K_{n}\right)=h\left(x_{n}\right)(\forall n \in \mathbb{N})$, so $h(T, K)=h(T)$ (by (5.2)). That is, $K$ is just the subset we need.

Remark 5.10. Let $(X, T)$ be a TDS with positive entropy. We can't require the subset $K$ in Theorem 5.9 to be $T$-invariant, since the topological entropy of any homeomorphism on a compact countable space is zero. Meanwhile, Example 5.3 implies that we can't require $K$ to have only one limit point.

Now let $\pi:(X, T) \rightarrow(Y, S)$ be a factor map such that $\pi^{-1}(y)$ is countable for each $y \in Y$. Is it possible that $h(T)>h(S)$ ? The answer is negative, since if $h(T)>h(S)$, then there is $y \in Y$ such that $\pi^{-1}(y)$ contains an uncountable scrambled set; see [22]. 
Now we give two interesting examples stated in Example [5.11. Let $(X, T)$ be a TDS. By Corollary 5.4 one has

$$
\begin{gathered}
E_{u p}(X, T) \supseteq \bigcup\left\{\operatorname{Supp}(\mu): \mu \in \mathcal{M}^{e}(X, T), h_{\mu}(T)>0\right\}, \\
E_{u p}^{f}(X, T) \supseteq \bigcup\left\{\operatorname{Supp}(\mu): \mu \in \mathcal{M}^{e}(X, T), h_{\mu}(T)=h(T)>0\right\} .
\end{gathered}
$$

The first example in Example 5.11 shows that the converse of the above inclusions need not hold. In fact, for this example $E_{u p}^{f}(X, T) \backslash \operatorname{Supp}(X, T) \neq \emptyset$, where $\operatorname{Supp}(X, T)$ is the union of the supports of all invariant measures. Since $E_{p}(X, T)$ is closed and $T$-invariant, we may consider $E_{p}\left(E_{p}(X, T), T\right)$. The same example tells us that $E_{p}(X, T)$ may contain isolated points and so $E_{p}\left(E_{p}(X, T), T\right) \subsetneq E_{p}(X, T)$. Moreover, we have a $\operatorname{TDS}(X, T)$ with $E_{p}(X, T)=X$ and $\operatorname{Supp}(X, T) \neq X$.

Example 5.11. We have the following examples:

1: There exists a $\operatorname{TDS}(X, T)$ such that $E_{u p}^{f}(X, T) \backslash \operatorname{Supp}(X, T) \neq \emptyset$ and $E_{p}(X, T)$ contains isolated points, which implies $E_{p}\left(E_{p}(X, T), T\right) \subsetneq$ $E_{p}(X, T)$.

2: There exists a $\operatorname{TDS}(X, T)$ such that $E_{p}(X, T)=X$ and $\operatorname{Supp}(X, T) \neq X$.

Proof. 1. Let $\left\{x^{i}\right\}_{i \in \mathbb{Z}_{+}}$be a closed subset of $\{1,2\}^{\mathbb{Z}}$ such that $\lim x^{i}=x^{0}=$ $(\cdots, 1 ; 1, \cdots)$ and $h\left(\sigma,\left\{x^{i}\right\}_{i \in \mathbb{Z}_{+}}\right)=\log 2$ (by Remark 5.5 and Corollary [5.8, such a subset exists). For each $i \in \mathbb{Z}_{+}$let $y^{i}=\left(\cdots, 0 ; x^{i}(1), x^{i}(2), \cdots\right)$ and $\overline{0} \doteq$ $(\cdots, 0 ; 0, \cdots)$. Set

$$
X=\{1,2\}^{\mathbb{Z}} \cup\{\overline{0}\} \cup\left\{\sigma^{n}\left(y^{i}\right): n \in \mathbb{Z}, i \in \mathbb{Z}_{+}\right\} .
$$

Clearly, $X \subseteq\{0,1,2\}^{\mathbb{Z}}$ is closed and invariant under the shift $\sigma$. It's not hard to show that $E_{p}(X, \sigma)=E_{u p}^{f}(X, \sigma)=\{1,2\}^{\mathbb{Z}} \cup\{\overline{0}\} \cup\left\{\sigma^{n} y^{0}: n \in \mathbb{Z}\right\}$, whereas $\operatorname{Supp}(X, \sigma)=\{1,2\}^{\mathbb{Z}} \cup\{\overline{0}\} \subsetneq X$. At the same time, it is easy to see that $E_{p}\left(E_{p}(X, \sigma), \sigma\right)=\{1,2\}^{\mathbb{Z}} \subsetneq E_{p}(X, \sigma)$ and $\sigma^{n} y^{0}$ is an isolated point of $E_{p}(X, \sigma)$ for each $n \in \mathbb{Z}$.

2. Let $X \subseteq\{0,1,2\}^{\mathbb{Z}}$ with $\left(x_{i}\right)_{-\infty}^{\infty} \in X$ iff $\left(x_{i}\right)_{-\infty}^{\infty} \in\{1,2\}^{\mathbb{Z}}$ or $\left(x_{i}\right)_{-\infty}^{\infty}=\overline{0}$ or there exists $j \in \mathbb{Z}$ such that $x_{k}=0$ for $k \leq j$ and $x_{k} \in\{1,2\}$ for $k>j$. Clearly, $X \subseteq\{0,1,2\}^{\mathbb{Z}}$ is closed and invariant under the shift $\sigma$. It's not hard to show that $E_{u p}^{f}(X, \sigma)=X$, whereas $\operatorname{Supp}(X, \sigma)=\{1,2\}^{\mathbb{Z}} \cup\{\overline{0}\} \subsetneq X$.

To end the section we give two remarks which state that some results obtained in the previous sections hold for more general settings.

Remark 5.12. Let $Z$ be a compact metric space and $R: Z \rightarrow Z$ a continuous map. Similarly, following Bowen's definition we can also introduce separated and spanning subsets and define the topological entropy of $(Z, R)$ (we use the same notations with TDS). Moreover, along the line of sections 4 and 5 , we can prove a result analogous with Theorem 5.9 (we skip the proof, since it is just a repetition). Precisely, if $(Z, R)$ has positive topological entropy, then: (1) there exists a countable closed subset $K_{1} \subseteq Z$ such that $h\left(R, K_{1}\right)>0$ and $K_{1}$ contains only one limit point; (2) there exists a countable closed subset $K_{2} \subseteq Z$ such that $h\left(R, K_{2}\right)=h(R)>0$.

Remark 5.13. Let $(X, T)$ be a TDS and $E$ a closed subset of $X$. For each $\epsilon>0$ and $x \in E$ define $h(x, \epsilon, E)=\inf \{r(d, T, \epsilon, K): K$ is a closed neighborhood of $x$ in $E\}$. 
Let $h(x, E)=\lim _{\epsilon \rightarrow 0+} h(x, \epsilon, E)$. Then using the same methods we could show

1: $h(x, \epsilon, E)$ is u.s.c. and $\sup _{x \in E} h(x, E)=h(T, E)$.

2: If $E^{\prime}$ is a countable closed subset of $E$ with a unique limit point $x$, then $h\left(T, E^{\prime}\right) \leq h(x, E)$.

3: For each $x \in E$ there is a countable closed subset $E_{x}$ of $E$ with a unique limit point $x$ such that $h\left(T, E_{x}\right)=h(x, E)$.

4: There is a countable closed subset $E^{\prime} \subseteq E$ with $h\left(T, E^{\prime}\right)=h(T, E)$. Moreover, $E^{\prime}$ can be chosen such that the set of limit points of $E^{\prime}$ has at most one limit point, and $E^{\prime}$ has a unique limit point iff there is $x \in E$ with $h(x, E)=h(T, E)$.

\section{C-Entropy point in topological AND MEASURE-THEORETICAL SETTINGS}

Different from the previous sections, in this section we introduce the notions of C-entropy point both in topological and measure-theoretical settings along the line of entropy pair $([2],[5])$, tuple $([15])$, sequence and set $([9])$. We show that each C-entropy point is an entropy point, and the set of C-entropy points is a union of sets of C-entropy points for a measure over all invariant measures. Some other properties of C-entropy points are discussed.

Definition 6.1. Let $(X, T)$ be a TDS and $\mu \in \mathcal{M}(X, T)$.

(1) We say $x \in X$ is a $C$-entropy point if there exists $x^{\prime} \in X$ such that $\left(x, x^{\prime}\right) \in$ $E_{2}(X, T)$. Denote by $E_{1}(X, T)$ the set of all C-entropy points.

(2) We say $x \in X$ is a $C$-entropy point for $\mu$ if there exists $x^{\prime} \in X$ such that $\left(x, x^{\prime}\right) \in E_{2}^{\mu}(X, T)$. Denote by $E_{1}^{\mu}(X, T)$ the set of all C-entropy points for $\mu$.

It is clear that both $E_{1}(X, T)$ and $E_{1}^{\mu}(X, T)$ are $T$-invariant (as both $E_{2}(X, T)$ and $E_{2}^{\mu}(X, T)$ are $T \times T$-invariant), $E_{1}(X, T) \neq \emptyset$ iff $h(T)>0$ and $E_{1}^{\mu}(X, T) \neq \emptyset$ iff $h_{\mu}(T)>0$. Since $E_{1}(X, T)=p_{2}\left(E_{2}(X, T)\right)\left(\right.$ resp. $\left.E_{1}^{\mu}(X, T)=p_{2}\left(E_{2}^{\mu}(X, T)\right)\right)$, we have

Proposition 6.2. Let $(X, T)$ be a TDS. Then

1: $\forall \mu \in \mathcal{M}(X, T), E_{1}^{\mu}(X, T) \subseteq E_{1}(X, T) \subseteq E_{p}(X, T)$.

2: There exists $\mu_{0} \in \mathcal{M}(X, T)$ such that $E_{1}^{\mu_{0}}(X, T)=E_{1}(X, T)$.

3: $E_{1}(X, T) \subseteq X$ is an $F_{\sigma}$ subset.

4: $\forall \mu \in \mathcal{M}(X, T), E_{1}^{\mu}(X, T) \subseteq X$ is an $F_{\sigma}$ subset.

Proof. Parts 1 and 2 follow directly from Proposition 3.1 and Theorem 3.4. By part 2, part 3 follows from part 4 . Now we are going to prove part 4 .

Say $d$ is a metric on $X$. Since

$$
E_{2}^{\mu}(X, T)=\operatorname{Supp}\left(\lambda_{2}(\mu)\right) \backslash \Delta_{2}(X)=\bigcup_{n \geq 1} \operatorname{Supp}\left(\lambda_{2}(\mu)\right) \backslash\left\{\left(x_{1}, x_{2}\right): d\left(x_{1}, x_{2}\right)<\frac{1}{n}\right\},
$$

where $\operatorname{Supp}\left(\lambda_{2}(\mu)\right) \backslash\left\{\left(x_{1}, x_{2}\right): d\left(x_{1}, x_{2}\right)<\frac{1}{n}\right\} \subseteq X^{(2)}$ is closed $(\forall n \in \mathbb{N}), E_{1}^{\mu}(X, T)$ $=p_{2}\left(E_{2}^{\mu}(X, T)\right)$ is the union of a countably many closed subsets. This ends the proof. 
As a direct corollary of Proposition 3.2 one has

Proposition 6.3. Let $(X, T)$ be a TDS and $\mu \in \mathcal{M}(X, T)$. Say $\mu=\int_{\Omega} \mu_{\omega} d \eta(\omega)$ is the ergodic decomposition of $\mu$. Then

1: $E_{1}^{\mu_{\omega}}(X, T) \subseteq E_{1}^{\mu}(X, T)$ for $\eta$-a.e. $\omega \in \Omega$.

2: If $x \in E_{1}^{\mu}(X, T)$, then $\eta\left(\left\{\omega \in \Omega \mid V \cap E_{1}^{\mu_{\omega}}(X, T) \neq \emptyset\right\}\right)>0$ for any measurable neighborhood $V$ of $x$. Thus for an appropriate choice of $\Omega$ we have

$$
\overline{\bigcup\left\{E_{1}^{\mu_{\omega}}(X, T): \omega \in \Omega\right\}}=\overline{E_{1}^{\mu}(X, T)} .
$$

Now we investigate the topological entropy of $E_{1}(X, T)$ and $E_{1}^{\mu}(X, T)$.

Theorem 6.4. Let $(X, T)$ be a TDS and $\mu \in \mathcal{M}(X, T)$ satisfy $h_{\mu}(T)>0$. Then

1: If $\mu \in \mathcal{M}^{e}(X, T)$, then $\operatorname{Supp}(\mu)=\overline{E_{1}^{\mu}(X, T)}$.

2: $h\left(T, E_{1}^{\mu}(X, T)\right) \geq h_{\mu}(T)$.

Proof. 1. As $\operatorname{Supp}\left(\lambda_{2}(\mu)\right) \subseteq \operatorname{Supp}(\mu) \times \operatorname{Supp}(\mu)$, one has

$$
\overline{E_{1}^{\mu}(X, T)} \subseteq p_{2}\left(\operatorname{Supp}\left(\lambda_{2}(\mu)\right)\right) \text { (by Proposition 3.1) } \subseteq \operatorname{Supp}(\mu) .
$$

To show $\operatorname{Supp}(\mu) \subseteq \overline{E_{1}^{\mu}(X, T)}$, we follow the same discussion as in Theorem 3.5. Let $\mu=\int_{Y} \mu_{y} d \nu(y)$ be the disintegration of $\mu$ over its Pinsker factor. Then for $\nu$-a.e. $y \in Y, \operatorname{Supp}\left(\mu_{y}\right) \subseteq \overline{E_{1}^{\mu}(X, T)}$ and so $\operatorname{Supp}(\mu) \subseteq \overline{E_{1}^{\mu}(X, T)}$. That is, $\operatorname{Supp}(\mu)=\overline{E_{1}^{\mu}(X, T)}$.

2. It's not hard to check that $h(T, \bar{K})=h(T, K)$ for each $K \subseteq X$. Now for all $\mu \in \mathcal{M}^{e}(X, T)$ the conclusion follows from part 1. In general, by Proposition 6.3. $\Omega$ can be chosen appropriately such that $E_{1}^{\mu_{\omega}}(X, T) \subseteq E_{1}^{\mu}(X, T)(\forall \omega \in \Omega)$, where $\mu=\int_{\Omega} \mu_{\omega} d \eta(\omega)$ is the ergodic decomposition of $\mu$. Then

$$
h\left(T, E_{1}^{\mu}(X, T)\right) \geq \sup _{\omega \in \Omega} h\left(T, E_{1}^{\mu_{\omega}}(X, T)\right) \geq \sup _{\omega \in \Omega} h_{\mu_{\omega}}(T) .
$$

As $h_{\mu}(T)=\int_{\Omega} h_{\mu_{\omega}}(T) d \eta(\omega)$ (Theorem 8.4 in [21]), the right hand side in the inequality (6.1) is not less than $h_{\mu}(T)$, that is, $h\left(T, E_{1}^{\mu}(X, T)\right) \geq h_{\mu}(T)$. The proof is completed.

Theorem 6.5. Let $(X, T)$ be a TDS. Then

$$
\begin{aligned}
& \text { 1: } \overline{E_{1}(X, T)}=\overline{\bigcup\left\{S u p p(\mu): \mu \in \mathcal{M}^{e}(X, T), h_{\mu}(T)>0\right\}} . \\
& \text { 2: } h\left(T, E_{1}(X, T)\right)=h(T) \text {. }
\end{aligned}
$$

Proof. 1. The direction " $\supseteq$ " is clear (using Theorem 6.4). For the direction " $\subseteq$ ", by Proposition 6.2 there exists $\mu_{0} \in \mathcal{M}(X, T)$ such that $E_{1}(X, T)=E_{1}^{\mu_{0}}(X, T)$. Obviously, $E_{1}^{\mu_{0}}(X, T)$ is contained in $\overline{\left.\bigcup \operatorname{Supp}(\mu): \mu \in \mathcal{M}^{e}(X, T) \text { and } h_{\mu}(T)>0\right\}}$ (following from Proposition 6.3 (2) and Theorem 6.4(1)). This proves the equality.

2. It follows from Theorem 6.4 by applying (3.1).

Let $(X, T)$ be a TDS and $\mu \in \mathcal{M}(X, T)$ with $h_{\mu}(T)>0$. Say $\mu=\int_{\Omega} \mu_{\omega} d \eta(\omega)$ is the ergodic decomposition. Then $\Omega_{0}=\left\{\omega \in \Omega: h_{\mu_{\omega}}(T)>0\right\} \subseteq \Omega$ is measurable and $\eta\left(\Omega_{0}\right)>0$. In this case, set $\mu^{e} \doteq \frac{1}{\eta\left(\Omega_{0}\right)} \int_{\Omega_{0}} \mu_{\omega} d \eta(\omega)$; for example, $\mu^{e}=\mu$ if $\mu \in \mathcal{M}^{e}(X, T)$. Then $\mu=\eta\left(\Omega_{0}\right) \mu^{e}+\eta\left(\Omega \backslash \Omega_{0}\right) \mu^{r}$, where $\mu^{r}=$ $\frac{1}{\eta\left(\Omega \backslash \Omega_{0}\right)} \int_{\Omega \backslash \Omega_{0}} \mu_{\omega} d \eta(\omega)$ when $\eta\left(\Omega_{0}\right)<1$ and $\mu^{r}=\mu$ when $\eta\left(\Omega_{0}\right)=1$. Note that $\mu^{e}, \mu^{r} \in \mathcal{M}(X, T)$ are both determined completely by $\mu$. Now following similar discussions as in Theorem 6.4 it's not hard to obtain $\overline{E_{1}^{\mu}(X, T)}=\operatorname{Supp}\left(\mu^{e}\right)=$ 
$\bigcap_{\Omega^{*} \subset \Omega_{0}: \eta\left(\Omega^{*}\right)=\eta\left(\Omega_{0}\right)} \overline{\bigcup\left\{\operatorname{supp}\left(\mu_{\omega}\right): \omega \in \Omega^{*}\right\}}$ (for the second equality, see for example Proposition 5.1 in 9]).

Now using Propositions 3.1, 3.2 and 6.3 it's not hard to obtain

Proposition 6.6. Let $(X, T)$ be a TDS with $\mu \in \mathcal{M}(X, T)$. Then

1: $x \in \overline{E_{1}^{\mu}(X, T)}$ iff for each neighborhood $V$ of $x$ in $X$ one has $(V \times V) \cap$ $E_{2}^{\mu}(X, T) \neq \emptyset$.

2: $x \in \overline{E_{1}(X, T)}$ iff for each neighborhood $V$ of $x$ in $X$ one has $(V \times V) \cap$ $E_{2}(X, T) \neq \emptyset$.

Now let $2^{X}=\{K \subseteq X: K$ is closed and non-empty $\}$ be equipped with the Hausdorff topology. Then combining Proposition 6.6 with the results in 9 ] one has

Proposition 6.7. Let $(X, T)$ be a TDS. Then

1: For each $\mu \in \mathcal{M}(X, T)$ with $h_{\mu}(T)>0,\left\{K \in 2^{X}: K\right.$ is an entropy set for $\mu\} \cup\left\{\{x\}: x \in \overline{E_{1}^{\mu}(X, T)}\right\}$ is a closed non-empty subset of $2^{X}$.

2: If $(X, T)$ has positive entropy, then $\left\{K \in 2^{X}: K\right.$ is an entropy set $\} \cup\{\{x\}$ : $\left.x \in \overline{E_{1}(X, T)}\right\}$ is a closed non-empty subset of $2^{X}$.

Moreover, using the lift-up property of an entropy pair ([2], [5]), we have the liftup property of a C-entropy point in both settings. Recall that if $\pi:(X, T) \rightarrow(Y, S)$ is a factor map between TDSs, then

(1) $\forall\left(y_{i}\right)_{1}^{2} \in E_{2}(Y, S)$, there exists $\left(x_{i}\right)_{1}^{2} \in E_{2}(X, T)$ such that $\pi\left(x_{i}\right)=y_{i}, i=$ 1,2 .

(2) $\forall \mu \in \mathcal{M}(X, T), \forall\left(y_{i}\right)_{1}^{2} \in E_{2}^{\pi \mu}(Y, S)$, there exists $\left(x_{i}\right)_{1}^{2} \in E_{2}^{\mu}(X, T)$ such that $\pi\left(x_{i}\right)=y_{i}, i=1,2$.

An immediate consequence is

Proposition 6.8. Let $\pi:(X, T) \rightarrow(Y, S)$ be a factor map between TDSs. Then

1: $\forall y \in E_{1}(Y, S)$, there exists $x \in E_{1}(X, T)$ such that $\pi(x)=y$.

2: $\forall \mu \in \mathcal{M}(X, T), \forall y \in E_{1}^{\pi \mu}(Y, S)$, there exists $x \in E_{1}^{\mu}(X, T)$ such that $\pi(x)=y$.

To end the paper, we give two results about the structure of the set of C-entropy points.

It is natural to ask for a TDS, whose point will not be a C-entropy point. We have the following partial answer for a minimal TDS. To state the result we need some notations. Let $(X, T)$ be a TDS and $x_{0} \in X$. The proximal relation is defined by

$P=\left\{\left(x_{1}, x_{2}\right) \in X^{(2)}: \exists n_{i} \nearrow+\infty\right.$ and $x \in X$ such that $\left.\left(T^{n_{i}} x_{1}, T^{n_{i}} x_{2}\right) \rightarrow(x, x)\right\}$.

We call $x_{0}$ a distal point of $(X, T)$ if $\left\{x^{\prime} \in X:\left(x_{0}, x^{\prime}\right) \in P\right\}=\left\{x_{0}\right\}$. Denote by $D(X, T)$ the set of all distal points of the system. We have

Theorem 6.9. Let $(X, T)$ be a minimal TDS. Assume that $E_{1}(X, T) \neq X$. Then $X \backslash E_{1}(X, T)$ is a dense $G_{\delta}$ subset of $X$ and $D(X, T) \subseteq X \backslash E_{1}(X, T)$.

Proof. By Proposition 6.2 it is obvious that $X \backslash E_{1}(X, T)$ forms a $G_{\delta}$ subset of $X$, moreover, it is dense in $X$, as $(X, T)$ is minimal and $X \backslash E_{1}(X, T)$ is a $T$-invariant non-empty subset. Now we aim to show $D(X, T) \subseteq X \backslash E_{1}(X, T)$. 
Assume the contrary that there exists $x_{1} \in D(X, T)$ with $x_{1} \notin X \backslash E_{1}(X, T)$. This means $x_{1} \in E_{1}(X, T)$, and so there exists $x_{2} \in X$ such that $\left(x_{1}, x_{2}\right) \in$ $E_{2}(X, T)$.

Let $W=\overline{\left\{\left(T^{m} x_{1}, T^{m} x_{2}\right): m \in \mathbb{Z}_{+}\right\}}$. Then $W \subseteq E_{2}(X, T) \cup \Delta_{2}(X)$, since $E_{2}(X, T) \cup \Delta_{2}(X) \subseteq X^{(2)}$ is closed and $T \times T$-invariant, and $\left(x_{1}, x_{2}\right) \in E_{2}(X, T)$. Moreover, as $x_{1}$ is a distal point, we have $W \cap \Delta_{2}(X)=\emptyset$. Thus, $W \subseteq E_{2}(X, T)$. As $(X, T)$ is minimal, and $W$ is closed and $T \times T$-invariant, we have $p_{1}(W)=X$. This implies that $E_{1}(X, T)=p_{1}\left(E_{2}(X, T)\right)=X$, a contradiction, as we assume $E_{1}(X, T) \neq X$. This finishes the proof.

Call a TDS point distal if there is a distal point. It is well-known that for a minimal point distal TDS $(X, T), D(X, T)$ is a residual subset. Assume now that $(X, T)$ is a minimal TDS with positive entropy and $(Y, S)$ is a non-trivial distal TDS, and $\pi:(X, T) \rightarrow(Y, S)$ is almost one to one (for the existence of such an extension see [13]). It is not hard to show that $\left\{x \in X: \pi^{-1} \pi(x)=\{x\}\right\}=D(X, T)$ and $D(X, T) \subseteq X \backslash E_{1}(X, T)$. This is just the situation described in Theorem 6.9.

Moreover, in [11] Glasner constructed an example $(X, T)$ which is minimal point distal and $E_{2}(X, T) \cap D(X, T) \times D(X, T) \neq \emptyset$ (in fact, this example is a compact group extension of a TDS which is an almost one to one extension of a Kronecker system), in particular, $E_{1}(X, T) \cap D(X, T) \neq \emptyset$. Then applying Theorem 6.9 we know that for this example we have $E_{1}(X, T)=X$. For systems with this property, by Theorem 6.5 they admit invariant measures with full support.

We also have the following observation. Recall that a TDS $(X, T)$ has uniformly positive entropy (u.p.e., for short) if $E_{2}(X, T)=X^{(2)} \backslash \Delta_{2}(X)$, and it has completely positive entropy (c.p.e., for short) if any non-trivial factor of $(X, T)$ has positive entropy (see [1]). So the above example of Glasner is a minimal TDS with $E_{1}(X, T)=X$ which hasn't c.p.e. Examples with the properties can also be obtained in the following way.

Let $(Y, S)$ be any non-trivial u.p.e. TDS and $(Z, \Theta)$ any non-trivial minimal TDS with zero entropy. Set $(X, T)=(Y \times Z, S \times \Theta)$. Then Proposition 3.1 of [3. tells us that $E_{2}(X, T) \cup \Delta_{2}(X)=\left\{\left(\left(y_{1}, z\right),\left(y_{2}, z\right)\right): y_{1}, y_{2} \in Y, z \in Z\right\}$ and $(Z, \Theta)$ is the maximal zero entropy factor of $(X, T)$. Thus $(X, T)$ hasn't c.p.e. and $E_{1}(X, T)=X$. Note that $(Y, S)$ and $(Z, \Theta)$ are disjoint (by Proposition 6 of [2]), and so $(X, T)$ is minimal if $(Y, S)$ is minimal.

To finish the paper we ask the following questions. Our first question (asked by the referee) is

Question 6.10. Do there exist transitive non-minimal TDSs with properties stated in Example 3.9 and Example 5.3?

We have proved that for a TDS $(X, T), E_{1}(X, T) \subseteq E_{p}(X, T)$. It is clear that if $(X, T)$ has only finitely many ergodic measures, then $E_{1}(X, T) \subseteq E_{u p}(X, T)$ (using Corollary 5.4). However, it is not hard to construct an example $(X, T)$ by modifying Example 5.3 for which $\overline{E_{1}(X, T)} \subseteq E_{u p}(X, T)$ does not hold. Thus, our second question is

Question 6.11. Does $E_{1}(X, T) \subseteq E_{u p}(X, T)$ hold for any $\operatorname{TDS}(X, T)$ ?

\section{ACKNOWLEDGEMENT}

We would like to thank the referee for the careful reading and useful comments that resulted in substantial improvements to this paper. 


\section{REFERENCES}

[1] F. Blanchard, Fully positive topological entropy and topological mixing, Symbolic Dynamics and its Applications, AMS Contemporary Mathematics, 135(1992), 95-105. MR.1185082 (93k:58134)

[2] F. Blanchard, A disjointness theorem involving topological entropy, Bull. de la Soc. Math. de France, 121(1993), 465-478. MR1254749 (95e:54050)

[3] F. Blanchard and Y. Lacroix, Zero-entropy factors of topological flows, Proc. Amer. Math. Soc., 119(1993), 985-992. MR.1155593 (93m:54066)

[4] F. Blanchard, E. Glasner and B. Host, A variation on the variational principle and applications to entropy pairs, Ergodic Th. and Dynam. Sys., 17(1997), 29-43. MR1440766 (98k:54073)

[5] F. Blanchard, B. Host, A. Maass, S. Martínez and D. Rudolph, Entropy pairs for a measure, Ergodic Th. and Dynam. Sys., 15(1995), 621-632. MR.1346392 (96m:28024)

[6] F. Blanchard and W. Huang, Entropy sets, weak mixing sets and entropy capacity, to appear in Discrete and Continuous Dynamical Systems.

[7] M. Boyle and T. Downarowicz, The entropy theory of symbolic extensions, Invent. Math., 156 (2004), 119-161. MR2047659(2005d:37015)

[8] M. Brin and A. B. Katok, On local entropy, Geometric dynamics (Rio de Janeiro, 1981), 3038, Lecture Notes in Math. 1007, New York, Springer-Verlag (1983). MR730261 (85c:58063)

[9] D. Dou, X. Ye and G. H. Zhang, Entropy sequences and maximal entropy sets, Nonlinearity, 19(2006), 53-74. MR2191619 (2006i:37037)

[10] D. Fiebig, U. Fiebig and Z. Nitecki, Entropy and preimage sets, Ergodic Th. and Dynam. Sys., 23(2003), 1785-1806. MR2032488 (2004k:37025)

[11] E. Glasner, A simple characterization of the set of $\mu$-entropy pairs and applications, Israel Journal of Mathematics, 192(1997), 13-27. MR1489099 (98k:54076)

[12] E. Glasner, Ergodic Theory via Joinings, Mathematical Surveys and Monographs 101, American Mathematical Society, 2003. MR1958753 (2004c:37011)

[13] E. Glasner and B. Weiss, Topological entropy of extensions, Ergodic Theory and its connections with Harmonic Analysis, Cambridge University Press, 1995, 299-307. MR 1325706 (96b:54064)

[14] E. Glasner and B. Weiss, On the interplay between measurable and topological dynamics, Handbook of Dynamical systems, Vol. 1B, Hasselblatt and Katok, eds., Elsevier, Amsterdam, 2006, 597-648. MR2186250(2006i:37005)

[15] W. Huang and X. Ye, A local variational relation and applications, Israel Journal of Mathematics, 151(2006), 237-280. MR2214126 (2006k:37033)

[16] W. Huang, X. Ye and G. H. Zhang, A local variational principle for conditional entropy, Ergodic Th. and Dynam. Sys., 26(2006), 219-245. MR2201946 (2006j:37015)

[17] W. Huang, X. Ye and G. H. Zhang, Relative entropy tuples, relative u.p.e. and c.p.e. extensions, Israel Journal of Mathematics, 158(2007), 249-284.

[18] W. Huang, X. Ye and G. H. Zhang, Lowering topological entropy over subsets, In preparation.

[19] A. B. Katok, Lyapunov exponents, entropy and periodic points for diffeomorphisms, Publ. Math. I.H.E.S., 51(1980), 137-173.

[20] P. Romagnoli, A local variational principle for the topological entropy, Ergodic Th. and Dynam. Sys., 23(2003), 1601-1610. MR2018614 (2004i:37030)

[21] P. Walters, An Introduction to Ergodic Theory, Graduate Texts in Mathematics 79, SpringerVerlag, New York-Berlin, 1982. MR648108 (84e:28017)

[22] G. H. Zhang, Relative entropy, asymptotic pairs and chaos, Journal of London Mathematical Society (2), 73(2006), 157-172. MR2197376 (2006k:37035)

Department of Mathematics, University of Science and Technology of China, Hefei, Anhui, 230026, People's Republic of China

E-mail address: yexd@ustc.edu.cn

Department of Mathematics, University of Science and Technology of China, Hefei, Anhui, 230026, People's Republic of China

E-mail address: ghzhang@mail.ustc.edu.cn 